\title{
Study of Serum Lactate Dehydrogenase Levels in Critically Ill Dengue Patients Admitted in PICU
}

\author{
Anjum Equebal ${ }^{1}$, Sara Dhanawade ${ }^{2}$
}

\begin{abstract}
Background: Certain biochemical markers have been found useful in monitoring the progress and predicting the severity of dengue. Serum lactate dehydrogenase (LDH) is one such potential biomarker.

Aim and objective: To study the serum LDH levels in critically ill patients of dengue with warning signs and severe dengue and its relationship with severity of illness.

Materials and methods: Retrospective analytical study.

Results: Sixty patients with severe dengue and dengue with warning signs were included. Mean duration of fever at admission was $4 \pm 1.6$ days and the mean age was $9.6 \pm 5.06$ years. Male:female ratio was 1:1. Half the children were above 10 years. The median LDH value was $1,133.5$ IU [interquartile range (IQR) 640-1,732]. The mean LDH value was significantly higher in patients with severe dengue $(2,986.65 \pm 3,638.54)$ as compared to dengue with warning signs $(1,209.87 \pm 1,370.20)(p-0.047)$. The majority $(70 \%)$ of patients with severe dengue had LDH $>1,000$ IU and complications like severe bleeding, pleural effusion, ARDS, and shock were higher in this group. Mean hospital stay in patients with LDH $>1,000$ was $14.685 \pm 5.993$ days and in those with $\mathrm{LDH}<1,000$ was $8.732 \pm 3.312$ days $(p=0.000)$. Mean platelet count was significantly lower in severe dengue $(56,405.00 \pm 49,918.74)$ as compared to dengue with warning signs $(922,257.50 \pm 71,235.44)(p$ value 0.028$)$ and there was a weak negative correlation between LDH and platelet count which was non-significant ( $r=$ Karl Pearson coefficient $-0.055 ; p$ value 0.676$)$. The case fatality rate was $9 \%$. The mean $\operatorname{LDH}(4,783 \pm 5,131)$ in non-survivors was much higher than survivors $(1,531.1 \pm 1,986)$ though this was not statistically significant.

Conclusion: Serum LDH level at admission was significantly raised in severe dengue as compared to dengue with warning signs. Similarly, mean $\mathrm{LDH}$ values were higher in survivors as compared to non-survivors. There was a weak negative correlation between LDH and platelet count.

Clinical significance: Lactate dehydrogenase can be used early in the disease to identify those who may progress to severe dengue and predict mortality. This will help optimize resource allocation and more effective care in a disease-endemic country like India.

Keywords: Dengue with warning signs, Lactate dehydrogenase, Severe dengue.

Pediatric Infectious Disease (2021): 10.5005/jp-journals-10081-1302
\end{abstract}

\section{INTRODUCTION}

Dengue fever is endemic in tropical countries. The clinical spectrum of dengue infection varies from no symptoms to severe dengue with shock. Nearly 100 million cases of dengue fever and between 250,000 and 500,000 cases of severe dengue are annually reported to the World Health Organization (WHO). Severe dengue is characterized by thrombocytopenia, spontaneous hemorrhages, and plasma leakage that can lead to shock. ${ }^{1}$

The name dengue was derived from the Swahili word for "bonebreaking fever" or the Spanish word for "the walk of a Dandie". 2 It is a self-limiting arboviral infection characterized by fever, rash, joint pains, nausea, vomiting, headache, and retro-orbital pain. As per the revised WHO criteria in 2009, the cases are classified as dengue without warning signs, dengue with warning signs, and severe dengue.

The disease severity may vary from mild fever which does not require hospitalization to severe disease with features of dengue hemorrhagic fever (DHF)/dengue shock syndrome (DSS) requiring intensive care management. ${ }^{3}$ The disease goes through three clinical stages like febrile phase, critical phase, and recovery phase. The febrile phase generally lasts for 2-10 days after which the patient may either enter the recovery phase or progress to a critical phase marked by defervescence and appearance of plasma leakage. Most complications of dengue occur in the above phase.
1,2Department of Paediatrics, Bharati Vidyapeeth (Deemed to be University) Medical College and Hospital, Sangli, Maharashtra, India

Corresponding Author: Sara Dhanawade, Department of Paediatrics, Bharati Vidyapeeth (Deemed to be University) Medical College and Hospital, Sangli, Maharashtra, India, Phone: +91 9113738459, e-mail: anjumkmc09@gmail.com

How to cite this article: Equebal A, Dhanawade S. Study of Serum Lactate Dehydrogenase Levels in Critically III Dengue Patients Admitted in PICU. Pediatr Inf Dis 2021;3(3):91-94.

Source of support: Nil

Conflict of interest: None

Despite the severity, the acute phase of dengue starts with a fever that is indistinguishable from other acute febrile infections.

Certain biochemical alterations in dengue may help in early diagnosis and predicting the severity. Studies have reported that patients with DHF have elevated levels of transaminases [aspartate aminotransferase (AST) and alanine aminotransferase (ALT)], amylase, lactate dehydrogenase (LDH), and creatine kinase (CK). ${ }^{4}$ The serum LDH levels have been reported to be increased in DF and still higher in DHF and DSS cases. ${ }^{5}$ It has been considered to be a diagnostic marker of dengue infection as well as an independent predictor of DHF. ${ }^{6}$ Sirikutt and Kalayanarooj observed mean LDH 
level to be $>500 \mathrm{IU}$ in dengue patients and $<500 \mathrm{IU}$ in non-dengue patients. Increasing levels of LDH were also seen toward the end of a febrile phase in DHF and DSS cases. ${ }^{7}$ Lactate dehydrogenase is an intracellular enzyme abundantly found in body tissues, e.g., muscles, liver, placenta, RBCs, and reticuloendothelial system. Its serum levels increase after cell injury. It has been evaluated as a prognostic marker of various inflammatory states like sepsis, infections, myocardial infarction, malignancies, and cardiopulmonary compromise. It is thought to be a marker of vascular permeability in immune-mediated lung injury. An early increase in LDH (three times the normal value) was found to be an independent predictor of DHF. ${ }^{8}$

Most of the studies on LDH in dengue infection have been in adults. This study was aimed to determine the admission serum LDH levels in patients of dengue with warning signs and severe dengue admitted to PICU and its relationship with severity.

\section{Materials and Methods}

This retrospective study was conducted in a tertiary care teaching hospital in Western Maharashtra from July 2019 to December 2019. The study was approved by the Institutional Ethical Committee.

Case records of all patients with a diagnosis of dengue fever based on a positive NS1 antigen and/or IgM ELISA test were screened. The cases with a discharge diagnosis of severe dengue or dengue with warning signs where serum LDH was done at admission were included for analysis. The details related to history, examination, and laboratory findings were noted. The complications, severity, and mortality were documented.

The WHO criteria for dengue with warning signs and severe dengue were used in the study. Accordingly, dengue with warning signs included the presence of the following:

- Abdominal pain or tenderness.

- Persistent vomiting.

- Clinical fluid accumulation.

- Mucosal bleed.

- Lethargy, restlessness.

- Liver enlargement $>2 \mathrm{~cm}$.

- Hematocrit increase with a rapid decrease in platelet count.

Severe dengue was defined as the presence of any of the following:

- Severe plasma leakage leading to (a) shock, (b) fluid accumulation with respiratory distress.

- Severe bleeding.

- Severe organ involvement (a) AST or ALT $\geq 1,000$, impaired consciousness, heart, and other organ involvement.

\section{Statistical Analysis}

Data were analyzed using descriptive statistics [mean and standard deviation, median and interquartile range (IQR) or frequency and percentage as deemed appropriate]. Categorical variables were analyzed using the Chi-square test. Continuous variables were analyzed using Student's $t$-test and the $r$ value was calculated by Pearson correlation coefficient.

\section{Results}

During the study period, 227 patients were admitted with dengue fever which comprised 94 cases of dengue with warning signs and severe dengue. Sixty cases admitted to PICU on whom serum LDH was done at admission were included in the study.

The mean duration of fever at admission was $4 \pm 1.6$ days and the mean age was $9.6 \pm 5.06$ years. Male:female ratio was 1:1. Half the children were above 10 years.

The median LDH value was 1,133.5 IU (IQR 640-1,732). The LDH values in the study cases ranged from 190 to 13,668 IU (mean$1,802)$. The majority (70\%) of severe dengue had LDH $>1,000 \mathrm{IU}$ as against $52 \%$ in dengue with warning signs $(p=0.012)$. The mean $\mathrm{LDH}$ value was significantly higher in patients with severe dengue $(2,986.65 \pm 3,638.54)$ as compared to dengue with warning signs $(1,209.87 \pm 1,370.20)(p=0.047)$

Shock and pleural effusion with respiratory distress were significantly higher in patients with $\mathrm{LDH}>1,000$. Other complications like severe bleeding, transaminitis, and ARDS were also more common in patients with LDH $>1,000$ IU, though this was not statistically significant. On checking the correlation between LDH values and platelet count, there was a negative but weak correlation between LDH and platelet count which was not significant $(r=\mathrm{Karl}$ Pearson coefficient $-0.055 ; p=0.676)$.

Mean hospital stay (14.685 \pm 5.993 days vs $8.732 \pm 3.312$ days) was significantly higher in patients with $\mathrm{LDH}>1,000$ as compared to those with $\mathrm{LDH}<1,000(p=0.000)$.

The case fatality rate was $9 \%$. The mean $\operatorname{LDH}(4,783 \pm 5,131)$ in non-survivors was much higher than survivors $(1,531 \pm 1,986)$ though this was not statistically significant. The causes of death included ARDS, refractory shock, DIC, and multi-organ dysfunction.

\section{Discussion}

Biochemical alterations detected after 48-96 hours of fever can predict a more severe form of dengue infection. This suggests that early pathogenic changes occur before complications develop. These potential biochemical markers may be used for monitoring illness and predicting severity. Serum LDH levels have been reported to be increased in dengue fever. However, there are limited studies in children evaluating its relationship to the severity of the disease.

Sixty cases admitted to PICU on whom serum LDH was done at admission were included in the study. Half (51\%) of the patients were above 10 years of age and the male:female ratio was 1:1. A similar finding was reported by Shankar and Prarthana. ${ }^{6}$ Out of 60 patients, 20 (33\%) had severe dengue and 40 (66\%) had dengue with warning signs (Tables 1 and 2).

Fever (100\%) was the most common presenting symptom followed by pleural effusion (41\%) and severe bleeding like severe gastrointestinal bleeding, hematuria in $11 \%$ of cases. Shankar and Prarthana reported fever in $100 \%$ cases and the bleeding tendency in $2 \%$ of cases. $^{6}$

The mean LDH in the study population was 1,802 IU. Our finding was similar to Sirikutt and Kalayanarooj ${ }^{7}$ where the mean LDH was $1,873 \mathrm{IU}$. Other studies by Perveen et al. ${ }^{8}$ and Liao et al. ${ }^{9}$

Table 1: Age-wise distribution

\begin{tabular}{lcc}
\hline Age (years) & Frequency & Percentage \\
\hline$<1$ & 8 & 13 \\
$1-5$ & 7 & 11 \\
$6-10$ & 14 & 23 \\
$11-17$ & 31 & 51 \\
Total & 60 & 100 \\
\hline
\end{tabular}


Study of Serum LDH Levels in Critically Ill Dengue Patients Admitted in PICU

Table 2: Distribution according to clinical manifestations

\begin{tabular}{lcc}
\hline & $n=60$ & Percentage \\
\hline Fever & 100 & 100 \\
Severe bleeding & 7 & 11 \\
Impaired consciousness & 4 & 6 \\
Pleural effusion & 25 & 41 \\
Shock & 13 & 21 \\
ARDS & 6 & 1 \\
\hline
\end{tabular}

Table 3: Distribution according to LDH levels

\begin{tabular}{lcll}
\hline & $<1,000(n=25)$ & $>1,000(n=35)$ & \\
Serum $L D H$ & $(\%)$ & $(\%)$ & $p$ value \\
\hline $\begin{array}{l}\text { Severe dengue }(n=20) \\
\begin{array}{l}\text { Dengue with warning } \\
\text { signs }(n=40)\end{array}\end{array}$ & $19(47)$ & $14(70)$ & 0.1952 \\
\hline
\end{tabular}

reported much lower mean LDH values (618.38 and 448.17) as compared to the present study. The mean LDH levels at admission were significantly higher in patients with a discharge diagnosis of severe dengue $(2,986.65 \pm 3,638.54)$ as compared to dengue with warning signs $(1,209.87 \pm 1,370.20)(p=0.047)$. Several authors have reported higher serum LDH in severe dengue as compared to non-severe dengue. ${ }^{1,6,8}$

A study by Sirikutt and Kalayanarooj ${ }^{7}$ from Thailand compared LDH levels in adult patients with dengue fever and other febrile illnesses. They did serial LDH estimations on enrolment day (day 0 ), day of leakage, and discharge day and found that LDH levels were higher in dengue patients and the LDH levels correlated with disease severity They reported a mean LDH level of 709.2 in DF, 1,873 in DHF, 654.5 in DSS, and 434 IU in non-dengue patients. The mean LDH levels were elevated in all groups of patients, but with different levels. They concluded that LDH $>500$ can be used to differentiate patients with dengue from non-dengue patients in the early febrile phase and if the level is 1,000 or more on day 0 it may be a predictor of severe dengue infection (Sirikutt). We also observed LDH levels $>1,000$ to be significantly associated with severe dengue ( $p=0.047$ ). The rise in LDH is attributed to skeletal muscle damage and/or liver damage.

Mean platelet count was significantly lower in severe dengue $(56,405.00 \pm 49,918.74)$ as compared to dengue with warning signs $(922,257.50 \pm 71,235.44)(p=0.028)$. The association between the severity of thrombocytopenia and the clinical presentation has been described in many earlier studies. ${ }^{10-13}$ However, there are no published reports on the correlation of LDH and platelet count. On checking the correlation between LDH values and platelet count, we observed a negative but weak correlation between LDH and platelet level which was not significant $(r=$ Karl Pearson coefficient -0.055 ; $p=0.676$ ). Studies on a larger number of patients are needed to evaluate whether LDH levels have a significant negative correlation with platelet count (Tables 3 and 4).

Mean hospital stay (14.685 \pm 5.993 vs $8.732 \pm 3.312$ days) was significantly higher in patients with $\mathrm{LDH}>1,000$ as compared to those with $\mathrm{LDH}<1,000$ ( $p=0.000)$. In a recent study, Mittal et al. evaluated LDH as an estimate of the duration of hospital stay in dengue cases and observed a correlation between LDH and time of discharge from the hospital. The authors calculated an estimate of the duration of hospital stay based on platelet counts and LDH where LDH levels on the day of least platelet count were used.
Table 4: Distribution according to complications and LDH level

\begin{tabular}{llcl}
\hline & $\begin{array}{l}\text { LDH<1,000 } \\
(n=25)(\%)\end{array}$ & $\begin{array}{c}\text { LDH>1,000 } \\
(n=35)(\%)\end{array}$ & p value \\
\hline $\begin{array}{l}\text { Severe bleeding } \\
\text { AST or ALT }>1,000\end{array}$ & $1(4)$ & $6(17)$ & 0.118 \\
$\begin{array}{l}\text { Impaired } \\
\text { consciousness }\end{array}$ & $1(4)$ & $5(14)$ & 0.19 \\
$\begin{array}{l}\text { Pleural effusion with } \\
\text { respiratory distress }\end{array}$ & $6(24)$ & $3(8)$ & 0.483 \\
$\begin{array}{l}\text { ARDS } \\
\text { Shock }\end{array}$ & $1(4)$ & $19(54)$ & 0.018 \\
Death & $4(16)$ & $14(40)$ & 0.19 \\
\hline
\end{tabular}

The discharge criteria in their study were platelet count showing a significant increase in three consecutive samples. ${ }^{3}$

Complications like shock and pleural effusion with respiratory distress were significantly higher in patients with $\mathrm{LDH}>1,000$. Mortality in the study was $9 \%$. Kamath et al. ${ }^{14}$ reported case fatality of $8.3 \%$ in severe dengue. The relatively high mortality in the present study was attributed to the late referral of cases. The mean LDH levels were higher in non-survivors although not statistically significant.

\section{Limitations}

The study is limited by a small sample size and its retrospective nature. We also did not have dynamic values of LDH in the different phases of illness which would have added valuable information.

\section{Conclusion}

Serum LDH at admission was significantly raised in severe dengue as compared to dengue with warning signs. It may be used early in the disease to identify those who may progress to severe dengue and help optimize resource allocation in a disease-endemic country like India. Lactate dehydrogenase had a weak negative but nonsignificant correlation with platelet count.

Serum LDH values should be prospectively evaluated in larger studies to assess their usefulness as an early predictor of severity and to determine the cut-off levels so that they can be included in the management guidelines.

\section{References}

1. Ravishankar K, Vinoth PN, Venkatramanan P. Biochemical and radiological markers as predictors of dengue severity in children admitted in a tertiary care hospital. Int J Contemp Pediatr 2015;2(4):311-316. DOI: 10.18203/2349-3291.ijcp20150923.

2. Cecilia D. Current status of dengue and chikungunya in India. WHO South-East Asia J Public Health 2014;3(1):22-27. DOI: 10.4103/22243151.206879.

3. Mittal SH, Mittal S, Govil T. Devising a prognostic predictive scale based on lactate dehydrogenase levels in dengue. Astrocyte 2015;2(2):69-71. DOI: 10.4103/2349-0977.172679.

4. Villar-Centeno LA, Díaz-Quijano FA, Martínez-Vega RA. Biochemical alterations as markers of dengue hemorrhagic fever. Am J Trop Med Hyg 2008;78(3):370-374. DOI: 10.4269/ajtmh.2008.78.370.

5. Ghosh B, Mandal S. Perspectives to DENV and GAS infections and associated serum LDH profiles. EC Microbiol 2018;14:612-618.

6. Shankar P, Prarthana B. Biochemical parameters (lactate dehydrogenase, serum albumin) as early predictor of severe dengue. 
Int J Contemp Pediatr 2017;4(2):464-469. DOI: 10.18203/2349-3291. ijcp20170690.

7. Sirikutt $P$, Kalayanarooj $S$. Serum lactate and lactate dehydrogenase as parameters for the prediction of dengue severity. J Med Assoc Thai 2014;97:S220-S231.

8. Perveen $\mathrm{S}$. Relationship between serum lactate dehydrogenase levels and dengue severity. J Rawalpindi Med Coll 2017;21(1):9-12.

9. Liao B, Tang Y, Hu F, et al. Serum levels of soluble vascular cell adhesion molecules may correlate with the severity of dengue virus- 1 infection in adults. Emerg Microbes Infect 2015;4(4):e24-e27. DOI: 10.1038/ emi.2015.24.

10. Jayashree K, Manasa GC, Pallavi P, et al. Evaluation of platelets as predictive parameters in dengue fever. Indian J Hematol Blood Transfus 2011;27(3):127-130. DOI: 10.1007/s12288-011-0075-1.
11. Shinde PA. Evaluation of platelet count in pediatric patients with dengue fever: a hospital based study. Int J Contemp Med Res 2017;4(5):1175-1177.

12. Navya BN, Patil S, Kariappa TM. Role of platelet parameters in dengue positive cases - an observational study. Int J Health Sci Res 2016;6(6):74-78.

13. Jayanthi HK, Tulasi SK. Correlation study between platelet count, leukocyte count, nonhemorrhagic complications, and duration of hospital stay in dengue fever with thrombocytopenia. J Family Med Prim Care 2016;5(1):120. DOI: 10.4103/2249-4863.184635.

14. Kamath SR, Ranjit S. Clinical features, complications and atypical manifestations of children with severe forms of dengue hemorrhagic fever in South India. Indian J Pediatr 2006;73(10):889-895. DOI: 10.1007/BF02859281. 